\title{
Vehicle Number Plate Recognition System: A Literature Review and Implementation using Template Matching
}

\author{
Aniruddh Puranic \\ Undergraduate Student \\ Dept. of CSE, BMSCE \\ Bangalore - 560019, India
}

\author{
Deepak K. T. \\ Undergraduate Student \\ Dept. of CSE, BMSCE \\ Bangalore - 560019, India
}

\author{
Umadevi V. \\ Associate Professor \\ Dept. of CSE, BMSCE \\ Bangalore - 560019, India
}

\begin{abstract}
The growing affluence of urban India has made the ownership of vehicles a necessity. This has resulted in an unexpected civic problem - that of traffic control and vehicle identification. Parking areas have become overstressed due to the growing numbers of vehicles on the roads today. The Automatic Number Plate Recognition System (ANPR) plays an important role in addressing these issues as its application ranges from parking admission to monitoring urban traffic and to tracking automobile thefts. There are numerous ANPR systems available today which are based on different methodologies. In this paper, we attempt to review the various techniques and their usage. The ANPR system has been implemented using template Matching and its accuracy was found to be $80.8 \%$ for Indian number plates.
\end{abstract}

\section{General Terms}

Character Recognition, Template Matching.

\section{Keywords}

Educational Institutions, Automatic Number Plate Recognition, Artificial Neural Networks, Template Matching.

\section{INTRODUCTION}

With the growing number of vehicles, finding a car park is a serious issue today for a large number of students and faculty at Educational Institutions. Most of the car parks are managed manually by security guards who do not keep a track of the number of vehicles entering and exiting the premises. Hence, the vehicle driver have to keep circling the car park in order to find a vacant slot leading to a wastage of time, not to mention the anxiety and frustration of the driver. The absence of the security guards may also lead to vehicle thefts.

In BMS College of Engineering, the problem is getting acute day by day due to the fact that the number of student enrolments is increasing year by year and a huge percentage of students and faculty own cars with the limited number of parking lots. It is not always possible to increase the parking area to accommodate the growing number of vehicles, therefore developing an efficient ANPR is the need of the hour. Over the last few years, the ANPR has become a useful approach for vehicle surveillance. Typically, an ANPR system consists of three main stages: 1) Number Plate Localization (NPL), 2) Character Segmentation (CS), and 3) Optical Character Recognition (OCR). The NPL stage is where the Number Plate is being detected. The Character Segmentation stage is an important pre-processing step before applying OCR, where each character from the detected Number Plate is segmented before recognition. In the last stage, characters are segmented from the Number Plate so that only useful information is retained for recognition where the image format will be converted into characters [1].Various research journals were consulted to find relevant information regarding ANPR based applications. ANPR systems are based on common approaches like Artificial Neural Network (ANN) [2,3],Probabilistic neural network (PNN) [4], Optical Character Recognition (OCR) [5,3], MATLAB[6], Configurable method[7], Sliding Concentrating window (SCW), Back-Propagation Neural Network [8], Support Vector Machine (SVM) [9], Inductive Learning [10], Regionbased, Color Segmentation [11] and Fuzzy-Based Algorithm [12], Scale Invariant Feature Transform (SIFT) [13].

In this paper, a system has been implemented using template matching for vehicle number plate recognition for BMS College of Engineering through video analysis of vehicle entry and exit into the college campus. The goal of the system is recognition of vehicle number plate using Template Matching. The remainder of the paper is organized as follows: existing methodologies are discussed in Section 2 of this paper; Section 3 describes proposed system and recognition mechanism.

\section{EXISTING METHODOLOGIES}

Computer vision and character recognition, algorithms for license plate recognition play an important role in video analysis of the number plate image. Therefore they form the core modules in any ANPR system. The system for automatic car license plate recognition includes a camera, a frame grabber, a computer, and custom designed software for image processing, analysis and recognition.

Vehicle identification has been an active research for over the last few years. A number of researches have been carried out to identify the type of vehicle such as a car, truck, scooter or motorcycle. In [14], Soble filter was used to address this issue to find the edges of the vehicle which in turn is applied to recognize the type of vehicle. The Contourlet Transform and Support Vector Machine (SVM) were used in [15] to find out the model of the vehicle. They showed numerical results on data set of about 70 pictures. However, they did not apply the technique to real-time video stream. In [16] monocular images are used for vehicle recognition. They applied canny edge detection to detect the presence of vehicle and SVM to recognize the vehicle. In [12], Maximum Average Correlation Height (MACH) filter and Log r-theta Mapping techniques were applied to recognize the type of vehicle irrespective of scale and rotation variation of vehicles. The MACH filter was used for detection of targets in cluttered environment. In [17], MACH was used to filter recognize the target to orientation invariance and they used $\log$ r-theta mapping to create inplane rotation and scale invariance while recognition. 
In [18], Optical Character Recognition (OCR) technique was used, which is a widely used technology which translates scanned images of printed text into machine encoded text. Here, an OCR algorithm based on feed-forward neural network is being proposed where two non-overlapping real character image data sets are used for training and testing the proposed neural network. The two non-overlapping image data sets were used to emulate real-world scenarios where the neural network will be subjected to. Artificial Neural Networks (ANN) are widely used intelligent computing architecture for pattern recognition. The most common used ANN is the multilayer feed-forward neural network which has a simple internal architecture that can classify inputs into a set of target categories. Typically, the works done in [19] and [20] use features extraction and binary pixels value to organize the inputs of neural network respectively, the former one is the most common used method for neural network, which can achieve good performance even under difficult environment. However, the feature extraction normally needs complex computation or multiple stages to extract features. Similar methods are present which use extra procedures during the training stage or after obtaining the results of neural network to handle difficult characters that belong to the sets of ambiguous characters. Additional training is used for the difficult characters (e.g. I/1, B/8 and O/D) and in [21] comparison of distinguishing parts of ambiguous characters is performed. The statistical classifiers can be divided into two sub-classes: single stage classifier and multistage classifier. In the work presented in [22], character features are extracted from the elastic mesh, and the entire address character string is taken as the object of study. This was tested using Japanese Number Plates and the Support Vector Machine (SVM) integration uses the features to recognize numbers, Kana (Japanese script), and the strings of characters that represent the area. The recognition rates for numbers, Kana and strings of characters are $99.5 \%, 98.6 \%$ and $97.8 \%$ respectively. In [23], a two-stage hybrid OCR system is presented to improve the recognition rate. It firstly uses four statistical subclassifiers to independently recognize the input character and then the results are combined using the Bayes' method [23].Secondly, if the recognized character from the first stage belong to the sets of ambiguous characters (e.g. I/1, B/8 and $\mathrm{O} / \mathrm{D})$, a structural stage is used for a further decision.

Common pattern matching technique is a simple technique for the recognition of single font and fixed size character, which is a suitable approach for ANPR systems. Incorrectly segmented characters from the character segmentation stage, where characters are not in the expected position or few of them are missed, may affect the OCR recognition. The neural networks and statistical classifiers, which give better outcome compare to common pattern matching technique, can overcome this problem because of their strong memory and self-adapting ability. However, in order to achieve good performance, large amount of samples and neurons are needed to obtain the neural networks. In [18], MATLAB has been used for the implementation of the algorithm on a PC equipped with a Dual Core $2.4 \mathrm{GHz}$ and $3 \mathrm{G}$ RAM. It has also been used to generate the weights of neural network. 6436 binary images with varying resolutions from the previous character segmentation stage were used. First of all, the binary images of the characters are resized to the same size. To choose the right size, several sizes of input images have been used for neural network training. High recognition rates can be achieved by using large character images but this will result in a more complex structure of the neural network as the number of weights will increase. The size corresponding to the best suitable result is used for the final neural network.

Each system proposed for vehicle identification and number plate recognition in the literature survey has its own pros and cons.

\section{PROPOSED SYSTEM}

The proposed system is focused to solve the two main problems prevalent in educational institutions, namely keeping a track of number and type of vehicles currently in the premise while also aiding owners with exact time their vehicle had left premise in case of thefts. The System consists of two major components, 1) A Video capturing source. 2) The application that is developed using MATLAB. The block diagram for the proposed system is as shown in the Figure 1.

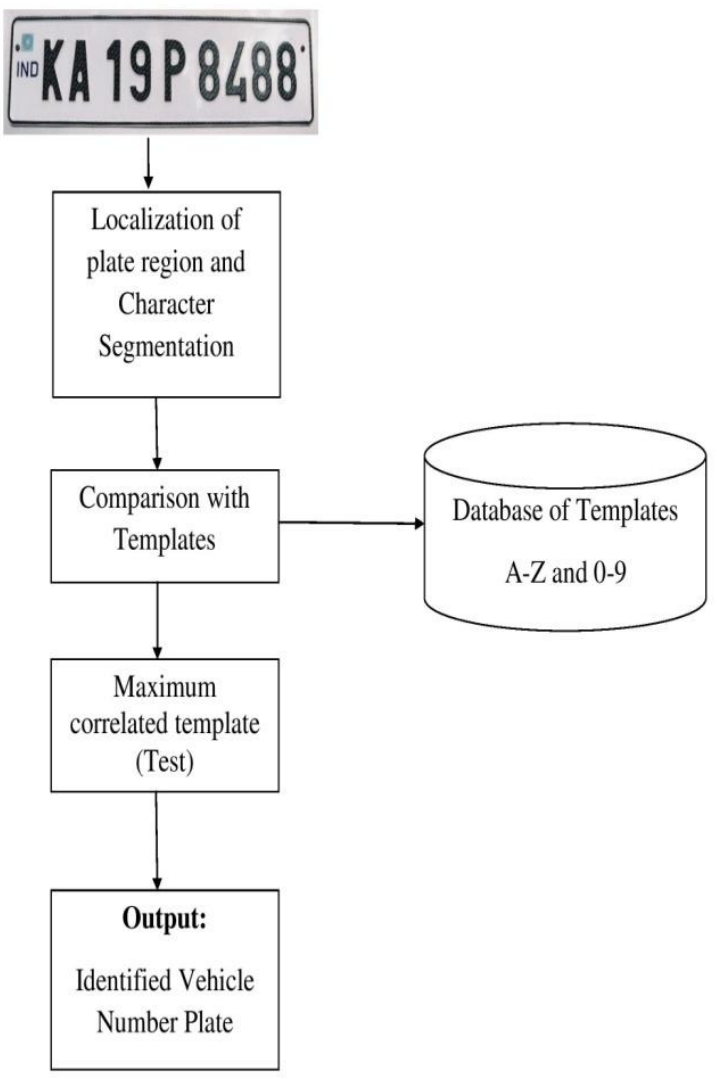

Figure 1: Block diagram of system for vehicle number plate recognition using Template Matching

The assumptions for the proposed system are: 1) Single Entry gate 2) Single Exit gate 3) Single lane traffic, further improving to double lane traffic. The video capturing source is placed at the entry of Institute which is capable of providing live feed of certain accuracy even in poor lighting. The video source is placed at a position which can capture the entire vehicle and the required frames consisting of a vehicle are consequently selected.

\subsection{Localization of number plate region and Character Segmentation}

The selected image is pre-processed by passing it over gray scale filter and edge detection is applied to isolate the region of interest, which is the number plate itself. A gray scale digital image is an image in which each pixel is quantized 
exclusively the shades of neutral gray, varying from black at the weakest intensity to white at the strongest intensity. The obtained gray image is then binarized, that is, it is converted to logical matrix by giving the pixel values of 1 for white shade and 0 for black shade. Possible number plate areas in images are identified by observing sudden changes in contrast. Remaining areas are filtered out. The best possible number plate location is found out by comparing width by height factor of actual Indian number plates to the same factor of plate like areas found by this method. The system shows maximum efficiency when the width by height factor is set between 3 and 7 [13]. The gray level plate images are enhanced by applying contrast extension and median filtering techniques [2]. So, the contrast differences between images and the noises such as dirty regions in white background of the plate can be eliminated.

\subsubsection{Contrast Extension}

To extend the contrast of an image means equalization of the histogram of that image will be used. In other words, the contrast extension makes the image sharpen. The gray-level histogram of an image is the distribution of the gray level values in an image. The histogram equalization is a popular technique to improve the appearance of a poor contrasted image. The process of equalizing the histogram of an image consists of 4 steps: (i) Find the sum of the histogram values. (ii) Normalize these values dividing by the total number of pixels. (iii) Multiply these normalized values by the maximum gray-level value. (iv) Map the new gray level values.

\subsubsection{Median Filtering}

Median filter is used for eliminating the unwanted noisy regions. In this filtering method, the $3 \times 3$ matrices is passed around the image. The dimension of these matrices can be adjusted according to the noise level. The process of working is (i) one pixel is chosen as centre pixel of the $3 \times 3$ matrices, (ii) the surrounding pixels are assigned as neighborhood pixels, (iii) the sorting process are employed between these nine pixels from smaller to the bigger, (iv) the fifth element is assigned as median element, (v) these procedures are implemented to the all pixels in plate image.

\subsubsection{Character Segmentation}

The characters of the identified number plate region are segmented using Regionprops function of MATLAB to obtain bounding boxes for each of the characters. Regionprops function returns the smallest bounding box that contains a character. This method is used to obtain the bounding boxes of all characters in the number plate.

\subsection{OCR using Template Matching}

Template matching is one of the Character Recognition techniques. It is the process of finding the location of a subimage called a template, inside an image. Template matching involves determining similarities between a given template and windows of the same size in an image and identifying the window that produces the highest similarity measure. It works by pixel-by-pixel comparison of the image and the template for each possible displacement of the template. This process involves the use of a database of characters or templates. There exists a template for all possible input characters. Templates are created for each of the alphanumeric characters (from A-Z and 0-9) using 'Regular' font style. Figure 2 shows the templates for few of the alphanumeric characters.

\section{[o. Z<smiles>[1H]</smiles><smiles>[1H]</smiles> A
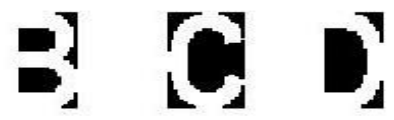

Figure 2: Template creation

For recognition to occur, the current input character is compared to each template to find either an exact match, or the template with the closest representation of the input character. It can capture the best position where the character is by moving standard template, thereby carry out the exact match. Moving template matching method is based on the template of target character, using the template of standard character to match the target character from eight directions of up, down, left, right, upper left, lower left, upper right, lower right. The results of template matching for character recognition on some of the Indian number plates taken from static images are shown in Table 1. The images of number plates used for template matching are shown in Figure 3.

Table 1: Results of Template Matching

\begin{tabular}{|c|c|c|c|}
\hline $\begin{array}{c}\text { Actual } \\
\text { Plate }\end{array}$ & $\begin{array}{c}\text { Predicted } \\
\text { Plate }\end{array}$ & $\begin{array}{c}\text { Mismatched } \\
\text { characters }\end{array}$ & Accuracy \\
\hline $\begin{array}{c}\text { DL 4C AF } \\
4943\end{array}$ & $\begin{array}{c}\text { DL 4C } \\
\text { AF 4943 }\end{array}$ & 0 & $100 \%$ \\
\hline $\begin{array}{c}\text { KA 19 P } \\
8488\end{array}$ & $\begin{array}{c}\text { KA 19 P } \\
\text { U4UU }\end{array}$ & 3 & $67 \%$ \\
\hline $\begin{array}{c}\text { MH 12 DE } \\
1433\end{array}$ & $\begin{array}{c}8812 \mathrm{DE} \\
1433\end{array}$ & 2 & $80 \%$ \\
\hline $\begin{array}{c}\text { WB 02 W } \\
6886\end{array}$ & $\begin{array}{c}\text { X8 02 X } \\
6886\end{array}$ & 2 & $80 \%$ \\
\hline
\end{tabular}

\section{4CAF 4943}
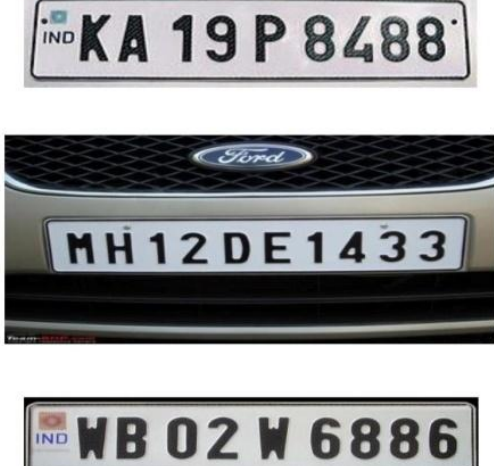

Figure 3: Images of Number Plates used for Template Matching 


\section{CONCLUSION AND FUTURE WORK}

In this work, existing methodologies and algorithms proposed in literature for Vehicle and Number Plate recognition were reviewed. Due to the unavailability of such an ANPR system off the shelf in tune with our requirements, it is our endeavor to customize an ANPR system for educational institution.

Template matching was implemented on number plates obtained from static images and an average accuracy of $80.8 \%$ was obtained. This accuracy can be improved greatly by positioning the camera suitably to capture the best frame and using two layers of neural networks. The implementation of the proposed system can be extended for the recognition of number plates of multiple vehicles in a single image frame by using multi-level genetic algorithms.

Also, a more sophisticated version of this system can be implemented by taking inputs from live video feed and selecting the best vehicle frame for classification of vehicle types and recognizing the number plates using neural networks.

\section{ACKNOWLEDGMENTS}

We are grateful to BMS College of Engineering for having provided us with the facilities needed for the successful completion of this Survey paper. The work reported in this paper is supported by the college through the TECHNICAL EDUCATION QUALITY IMPROVEMENT PROGRAMME [TEQIP-II] of the MHRD, Government of India. We thank Afraa Maryam, Undergraduate Student, Department of Computer Science and Engineering, BMSCE for being a vital and contributing member for completing this Survey paper.

\section{REFERENCES}

[1] Xiaojun Zhai, Faycal Bensaali, "Standard Definition ANPR System on FPGA and an Approach to Extend it to HD" in 2013 IEEE GCC Conference and exhibition, November 17-20, Doha, Qatar. pp.214

[2] H. Erdinc Kocer and K. Kursat Cevik, "Artificial neural networks based vehicle license plate recognition," Procedia Computer Science, vol. 3, pp. 1033-1037, 2011

[3] A Roy and D.P Ghoshal, "Number Plate Recognition for use in different countries using an improved segmentation," in 2nd National Conference on Emerging Trends and Applications in Computer Science(NCETACS), 2011, pp. 1-5

[4] Fikriye Öztürk and Figens Özen, "A New License Plate Recognition System Based on Probabilistic NeuralNetworks," Procedia Technology, vol. 1, pp. 124128,2012

[5] Anton Satria Prabuwono and Ariff Idris, "A Study of Car Park Control System Using Optical Character Recognition," in International Conference on Computer and Electrical Engineering, 2008, pp. 866-870

[6] Ch. Jaya Lakshmi, Dr. A. Jhansi Rani, Dr. K. Sri Ramakrishna, and M. Kanti Kiran, "A Novel Approach for Indian License Recognition System," International Journal of Advanced Engineering Sciences and Technologies, vol. 6, no. 1, pp. 10-14, 2011

[7] Jianbin Jiao, Qixiang Ye, and Qingming Huang, "A configurable method for multi-style license platerecognition," Pattern Recognition, vol. 42, no. 3, pp. 358-369, 2009
[8] Zhigang Zhang and Cong Wang, "The Research of Vehicle Plate Recognition Technical Based on BP Neural Network," AASRI Procedia, vol. 1, pp. 74-81, 2012

[9] Ying Wen, "An Algorithm for License Plate recognition Applied to Intelligent Transportation System", IEEE Transactions of Intelligent Transportation Systems. pp. $1-16,2011$

[10] Chirag Patel, Dipti Shah, Atul Patel," Automatic Number Plate Recognition System (ANPR): A Survey", International Journal of Computer Applications, 2013

[11] Yang Yang, Xuhui Gao, and Guowei Yang, "Study the Method of Vehicle License Locating Based on Color Segmentation," Procedia Engineering, vol. 15, pp. 13241329, 2011

[12] SaimaRafique, Mahboob Iqbal and Hafiz Adnan Habib, "Space Invariant Vehicle Recognition for Toll Plaza Monitoring and Auditing System", Multitopic Conference, 2009. INMIC 2009,IEEE 13th International, pp. 1-6

[13] Fajas F., Farhan Yousuf, Remya P. R., Adarsh P. Pavanan, Sajan Ambadiyil and Varsha Swaminathan, "Automatic Number Plate Recognition for Indian Standard Number Plates", Ultra Modern Telecommunications and Control Systems and Workshops (ICUMT), 2012 4th International Congress, pp. $1026-1028$

[14] Weihua Wang, "Reach on Sobel Operator for Vehicle Recognition, "International Joint Conference on Artificial Intelligence, pp.448-451, 2009

[15] Saeid Rahati, Reihaneh Morvejian, Ehsan M. Kazemi and Farhad M. Kazem "Vehicle Recognition Using Contourlet Transform and SVM," Proceedings of the Fifth International Conference on Information Technology, 2008

[16] M.A. Sotelo , J. Nuevo , L.M. Bergasa and M. Ocana, "Road Vehicle Recognition in Monocular Images," IEEE Symposium on Industrial Electronics, 2005

[17] Bone P, Young R, Chatwin C. "Position, rotation, scale, and orientation-invariant multiple object recognition from cluttered scenes," Opt Eng2006; 45:077203

[18] Xiaojun Zhai, Faycal Bensaali and Reza Sotudeh, "OCRBased Neural Network for ANPR" in IEEE, 2012. Pp1

[19] Y. Amit, D. Geman, and X. Fan, "A coarse-to-fine strategy formulticlass shape detection," IEEE Transactions on Pattern Analysis and Machine Intelligence, vol. 26, pp. 1606-1621, 2004

[20] C. Oz, and F. Ercal, "A Practical License Plate Recognition System for Real-Time Environments. Computational Intelligence and Bio-inspired System," Lecture Notes in Computer Science, vol. 3512/2005, pp.497-538, 2005

[21] S.L. Chang, L.S. Chen, Y.C. Chung and S.W. Chen, "Automatic license plate recognition," IEEE Transactions on Intelligent Transportation Systems, vol. 5, pp. 42-53, 2004

[22] Y. Wen, Y. Lu, J. Yan, Z. Zhou, von Deneen K.M. and P. Shi, "An Algorithm for License Plate Recognition Applied to Intelligent Transportation System," IEEE 
Transactions on Intelligent Transportation Systems, vol. 12 , pp. 830-845, 2011

[23] X. Pan, X. Ye and S. Zhang, "A hybrid method for robust car plate character recognition," presented at the IEEE International Conference on Systems, Man and Cybernetics, 2004

[24] C. Anantha Reddy, C. Shoba Bindu, "Multi-Level Genetic Algorithm for Recognizing Multiple License
Plates in a Single Image", Journal of Innovation in Computer Science and Engineering, 2015

[25] Anand Sumatilal Jain, Jayshree M. Kundargi, "Automatic Number Plate Recognition Using Artificial Neural Network", International Research Journal of Engineering and Technology (IRJET), 2015 